\title{
Previously treated versus untreated scoliosis: are results different?
}

\author{
Fabio Zaina ${ }^{1 *}$, Sabrina Donzelli', Monia Lusini ${ }^{1}$, Salvatore Minnella ${ }^{1}$, Stefano Negrini ${ }^{2}$ \\ From 11th International Conference on Conservative Management of Spinal Deformities - SOSORT 2014 \\ Annual Meeting \\ Wiesbaden, Germany. 8-10 May 2014
}

\section{Background}

Every day we see patients coming after some kind of brace treatment for adolescent idiopathic scoliosis (AIS). Literature is made mainly by studies about untreated patients, so we don't know what to expect when we start such a treatment.

\section{Aim}

The aim of the present study was to compare the results of previously treated scoliosis compared to untreated ones.

\section{Design}

Prospective observational controlled cohort study nested in a prospective database started in March 2003.

\section{Methods}

Inclusion criteria: patients that started a brace treatment at their first clinical evaluation at our institute between 2003 and 2009 for AIS, 12-15 years old, Risser 0-3.

Patients were divided in two groups, one of patients already treated with a brace (BRACE Group), and one never treated before (UNTREATED Group).

\section{Outcome measure}

The threshold of $5^{\circ}$ Cobb to define worsened, improved and stabilized curves was considered, average Cobb angle, ATR, TRACE (for aesthetic evaluation) Statistical analyses: Mean and SD were used for descriptive statistics of clinical and radiographic changes. Relative Risk of failure (RR), 95\% Confidence Interval (CI), Student's t, Kruskall Wallis, and chi square test were applied.

\section{Results}

268 patients were included (226 females), age $13.3( \pm 1)$.

BRACE Group: 108 (96 females), age $13.2( \pm 1)$, Cobb Angle $34 \pm 12^{\circ}$, ATR 9.6 \pm 0.4 , TRACE 5.7.

UNTREATED Group: 160 (130 females), age 13.2 $( \pm 1)$, Cobb Angle $33 \pm 11^{\circ}$ ATR $10.3 \pm 0.2^{\circ}$, TRACE 5 .

No differences among groups at first visit but for TRACE $(\mathrm{p}<0.05)$.

$49.38 \%$ of patients improved in UNTREATED, 43.13 stable, 7.50 worsened vs $35.19,52.78$ and 12.04 for BRACE $(\mathrm{p}=0.06)$. The Cobb angle was 28.9 vs $30.1(\mathrm{p}=0.06)$. The RR of failure for BRACE was 1.6 (IC95\%0.86-2.35). No differences among groups for TRACE and ATR. Drop out had results similar to the completers (NS).

\section{Conclusions}

Average clinical and radiological parameters improved in both groups. In the UNTREATED group results were slightly better even not significant, probably for the low statistical power. This study demonstrate that with a good treatment it' possible to achieve good clinical results even in already treated patients.

Authors' details

${ }^{1}$ ISICO, Milan, Italy. ${ }^{2}$ Don Gnocchi - Brescia University, Brescia, Italy.

Published: 4 December 2014

\section{Reference}

1. Richards BS, Bernstein RM, D'Amato CR, Thompson GH: Standardization of criteria for adolescent idiopathic scoliosis brace studies: SRS Committee on Bracing and Nonoperative Management. Spine (Phila Pa 1976); 2005:30(18):2068-2075.

doi:10.1186/1748-7161-9-S1-O21

Cite this article as: Zaina et al:: Previously treated versus untreated scoliosis: are results different? Scoliosis 2014 9(Suppl 1):O21. 